\title{
Quality improvement project using a care bundle approach on the management of the immediate discharge document (IDD) within a single general practice
}

Caroline Anderson, Duncan McNab

NHS GGC

\begin{abstract}
The Scottish Patient Safety Programme in Primary Care (SPSP-PC) aims to improve the medicines reconciliation process in primary care to help reduce the number of adverse events causing avoidable harm. [1] The aim of this project is to improve the process for handling Immediate Discharge Documents (IDDs) in a single practice and develop a protocol using the care bundle approach. The care bundle consisted of:
\end{abstract}

1. Medicines reconciled and repeat prescription updated

2. Follow up documented

3. Diagnosis coded

4. Were all actions completed?

A baseline audit was performed followed by three PDSA cycles. Interventions included: education at each cycle, decision that all Doctors would use the Medicines Reconciliation Polypharmacy LES template and constructing an electronic checklist for the care bundle. Compliance with the care bundle rose from $20 \%$ in the baseline measurement to $100 \%$ in PDSA cycles two and three.

In conclusion, a protocol was developed for the processing of IDDs utilizing the care bundle approach with an electronic checklist, resulting in an improvement in the practice management of IDDs.

\section{Problem}

The Scottish Patient Safety Programme in Primary Care (SPSP$P C)$ aims to reduce the number of adverse events causing avoidable harm to patients in this setting. Safer medicine management across the primary-secondary care interface is a core programme work stream. The programme encourages the development of reliable, safe systems in general practices for reconciling medicines following patient discharge.[1] A large number of readmissions are related to medication and many of these are thought to be avoidable. [1]

In my training practice, although medicines reconciliation of immediate discharge documents (IDDs) was occurring, there was no agreed protocol in place for the processing of IDDs. This has resulted in a lack of consistency in the medicines reconciliation process.

\section{Background}

Providing a discharge summary is one of the duties of a Doctor outlined by the General Medical Council. [2] It facilitates sharing of information from secondary to primary care and symbolises the transfer of care back to the patient's General Practitioner.[3] With emphasis on secondary care providing better quality IDDs it is imperative primary care acknowledge and utilize this information to maintain accurate, up to date patient records. A previously developed care bundle for the management of IDDs was recommended by SPSP. [1][Box 1]

The project was carried out in an urban training practice with a list size of approximately 5200 patients. With high numbers of patients over the age of 65 with significant multi-morbidity, polypharmacy, and communication difficulties such as visual, auditory, and memory problems; medicines reconciliation was considered a valuable area to study. Egton Medical Information Systems (EMIS) electronic patient records were used in the practice. [4]

There have been multiple recent changes in the practice: including staff shortage through ill health, the imminent merger with another practice, and the employment of a long-term locum doctor. With these changes it is essential to ensure the practice deals with hospital discharge letters appropriately; ideally all using the same process. This is particularly important for a training practice with the regular rotation of registrars and employment of locum staff. My aim for this project is the development of a protocol for the processing of IDDs; building on the existing Medicines Reconciliation 
Polypharmacy LES template.

Existing system

IDDs were distributed by a document workflow system (Docman) to the Doctors available that day. Some Doctors invited the patient to attend to discuss their admission and medications and process the IDD at that time; some would complete this over the phone and at times the medications would be altered without discussion with the patient. Under a Local Enhanced Polypharmacy Service the practice had access to a medicines reconciliation template, which was used by some Doctors. [Box 2] When the IDD was processed the patient's medical summary, repeat medications, and medicine reconciliation template were updated where deemed appropriate. This may have been carried out by doctors or instruction given to administrative staff. Administrative staff may also have communicated medication changes to the patient and/or pharmacy and follow up plans. Within the practice there were many processes for the handling of IDDs with no clear preferred system.

\section{Baseline measurement}

The baseline data was collected the week of 19th May 2014. All IDDs received were analysed.

There were five IDDs processed: three were over the age of sixtyfive years and two under sixty five years. $40 \%$ had medicines reconciled, $40 \%$ follow up documented and $20 \%$ had diagnosis coded.

Overall compliance with the care bundle was $20 \%$.

See supplementary file: ds4979.docx - "Box 1,2,3"

\section{Design}

A care bundle audit was employed using PDSA cycles to implement change. One week of data was examined at each cycle to determine improvement.

We decided to perform a baseline measurement followed by further data collections over a six month period utilizing plan do study act cycles; with the aim of achieving sustainable improvement. Patients were identified by looking through all the paperwork received during the designated week and the IDD documents identified. Individual EMIS [4] patient records were reviewed and the outcomes measured.

\section{Strategy}

A practice meeting was held with all doctors and staff to discuss current personal practice for the handling of IDDs and to achieve consensus on what should be included in a protocol. A number of areas were deemed essential. These were: medicines reconciliation completed with updating of repeat prescriptions, follow up documented, and diagnosis coded. A care bundle was then created.
When performing medicines reconciliation following hospital discharge the following should be done:

1. Medicines reconciled and repeat prescription updated

2. Follow up documented

3. Diagnosis coded

4. Were all actions completed?

All three actions had to be completed for each IDD.

Discussions around the diagnosis coding concluded that this was only necessary if the patient had suffered a new or significant diagnosis whilst in hospital.

The practice team decided to set standards at 50\% compliance with the bundle at one month, $75 \%$ at three months, and $80 \%$ at six months. These standards were chosen as this is a complex problem requiring changing established habits and behaviours.

\section{Results}

PDSA cycle 1

The results of the baseline measurement were discussed at the practice meeting and circulated to Doctors via email. The first intervention was the decision that all Doctors would use Medicines Reconciliation Polypharmacy LES template to process future IDDs. Further we decided to specifically look at patients over the age of sixty-five as they were more likely to experience hospital admission, multi-morbidity, polypharmacy, and adverse effects of medications.

Data was collected the week of 9th June 2014. Three IDDs were processed: $100 \%$ had medicines reconciled, $100 \%$ follow up documented and $67 \%$ had a diagnosis coded.

Overall compliance with the care bundle was $67 \%$.

PDSA cycle 2

Feedback on performance was discussed at the weekly practice meeting and emailed to the Doctors who could not attend. Educational intervention was provided at this point; stressing continued use of the Medicines Reconciliation Polypharmacy LES template. Further discussion focused on diagnosis coding as compliance was measured at $67 \%$ in PDSA cycle 1. We discussed accurate diagnosis coding and options for completing this; particularly asking administrative staff to complete coding via Docman as we felt they were more likely to record the appropriate read code.

Data was collected the week of 7th July 2014. Three IDDs were processed: $100 \%$ had medicines reconciled, $100 \%$ follow up documented, and $100 \%$ had diagnosis coded.

Overall compliance with the bundle was $100 \%$.

PDSA cycle 3 
Audit results were fed back to the team at the practice meeting and via email. Reflecting on compliance with the bundle it was felt that the short time between PDSA cycles and feedback of results was influencing doctors' behaviour positively. Consideration was then given to sustainability. A decision was made to amend the medicines reconciliation template. From previous PDSA cycles it was evident that coding a new or significant diagnosis was the area being missed most from the bundle. With the current medicines reconciliation template already encouraging Doctors to 'reconcile' medications we thought it would be helpful to incorporate diagnosis and follow up into the template. The team envisioned adding diagnosis with a tick box then a free type area and the same for follow up; matching the format for drug therapy discontinued in the existing template. The process proved more difficult than anticipated. Discussion with EMIS [4] and IT support highlighted the complexity of read coding, precluding the proposed changes. Time was spent considering how to improve the template to promote completion of the care bundle. A decision was made to add a tick box for new diagnosis and follow up to the template; creating an electronic checklist. To further promote completion of the bundle a forcing function was added to the checklist. The clinician was unable to close the template until they had ticked the box stating they had considered coding a diagnosis and documenting follow-up. [Box 3]

Final data collection was undertaken the week of 6th October 2014. Four IDDs were processed. 100\% medicines reconciled, 100\% follow up documented and $100 \%$ had diagnosis coded.

Overall compliance with the care bundle was $100 \%$.

The standard set for this quality improvement project was 50\% compliance with the bundle at one month, $75 \%$ at three months, and $80 \%$ at six months. Standards were exceeded at all three audit points. [Table 1]

See supplementary file: ds4978.docx - "Table 1"

\section{Lessons and limitations}

The aim of this project was to develop a practice policy for the processing of IDDs. Although numbers were small there was enough information to learn that systems for medicines reconciliation could be improved. There is now an electronic checklist in place incorporating the three actions of the care bundle; helping maintain accurate, up to date patient records.

The greatest improvement in compliance with the care bundle was between the baseline measurement and PDSA cycle one. This is explained by the decision that all Doctors would use the Medicines Reconciliation Polypharmacy LES template. Research has shown that using electronic templates can assist in sustaining standards. [5]

Further improvement in compliance with the bundle was seen in PDSA cycle two. During this PDSA cycle educational intervention was provided. This was in the form of weekly practice meetings and email communications stressing the importance of template use and regular feedback on performance. A Cochrane review recognises that audit and feedback can be effective in improving professional practice. [6]

In PDSA cycle three we took the decision to amend the existing template to incorporate diagnosis and follow up as these are two essential areas for accurate, up to date patient records.

Incorporating the forcing function ensures that the care bundle is fully complied with as long as Doctors continue to use the electronic checklist. Checklists have been shown to improve patient care and can allow Doctors to focus and address multiple issues for every patient, every time. [7] There are implications introducing tick boxes to a checklist. It cannot be assumed from a tick being placed in the diagnosis or follow up box that the corresponding information is then accurately coded or recorded in EMIS. [4]

In the first PDSA cycle it was decided to include patients over the age of sixty-five only. The team appreciated the importance of medicines reconciliation in this population due to their risk of hospital admission, polypharmacy and adverse effects of medications. However this resulted in small numbers of patients. Despite small numbers there is evidence of improvement in compliance with the care bundle. Using two weeks of data at each cycle would have given larger numbers to help determine compliance with the bundle.

The proximity of data collections and interventions, particularly educational, explains the Doctors change in behaviour. It would be worthwhile completing a further data collection around twelve months to examine for sustained improvement.

A further limitation of this project is the lack of patient involvement in the processing of IDDs. One of the measures in the SPSP Medicines Reconciliation care bundle is the discussion of any changes to the medications with the patient or their representative within seven days of receipt. [Box 1] There is, as yet, no standard procedure for performing this task within the practice and various methods are used. This is due to difficulty agreeing on the best approach. This area was not studied in this project but we have discussed how to incorporate patient involvement into the reconciliation process. Suggestions have included appointing IDDs to doctors on the day in individual slot notes with allocated time to complete the medicines reconciliation checklist and telephone the patient.

\section{Conclusion}

This project has utilized the care bundle approach to improve the processing of IDDs in a training practice, with consideration given to patient safety; prescribing, and maintaining accurate patient records. Simple measures such as team communication to raise awareness of practice policies and protocols can be effective in improving their use. Amending existing templates and using checklists can further add to the safety of processing patient documents.

\section{References}




\section{BMJ Quality Improvement Reports}

1. Scottish Patient Safety Programme. Medicines Reconciliation Care Bundle.

http://www.scottishpatientsafetyprogramme.scot.nhs.uk/prog rammes/primary-care/safer-medicines/medicinesreconciliation-care-bundle (accessed 20 January 2015)

2. General Medical Council: Good medical practice (2013). http://www.gmc-uk.org/static/documents/content/GMP 2013 .pdf 51447599.pdf (accessed 20 January 2015)

3. SIGN guidelines. SIGN Discharge Document (June 2012). Accessed http://www.sign.ac.uk/pdf/sign128.pdf (accessed 20 January 2015)

4. Egton Medical Information Systems (EMIS). http://www.emis-online.com/copyright (accessed 10 February 2015)

5. Swinglehurst D, Greenhalgh T, Roberts C. Computer templates in chronic disease management: ethnographic case study in general practice. BMJ Open 2012 2(6)

6. Jamtvedt G, Young JM, Kristoffersen DT, O'Brien MA, Oxman AD. Audit and feedback: effects on professional practice and health care outcomes. Cochrane Database Syst Rev. 2006;2:CD000259

7. Weiser G, Haynes A, Lashoher B, Dziekan G, Boorman D, Berry R, Gawande A. Perspectives in quality: designing the WHO Surgical Safety Checklist. Int J Qual Health Care 2010;22(5):365-70.

\section{Declaration of interests}

Nothing to declare.

\section{Acknowledgements}

Dr Alan Finney, Dr Linsey Semple, Mr Ryan Addison

\section{Ethical approval}

We used the following criteria for determining if improvement activities require ethics review. Policy criterion: The work is primarily intended to improve patient care and not provide generalisable knowledge in a field of inquiry. Explanation: The work reported here meets this criterion because processing patient IDDs is an essential activity undertaken in primary care and we sought only to evaluate the improvements in processing of IDDs by audit and feedback to medical staff. 